\title{
Mudanças dimensionais dos arcos dentários em crianças entre 3 e 6 anos de idade*
}

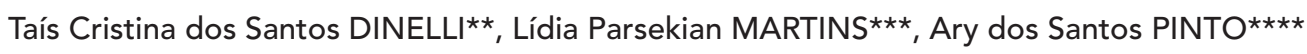

Resumo

Para o presente trabalho, 235 crianças pertencentes às creches da Prefeitura Municipal de Araraquara foram avaliadas e moldadas com um dispositivo confeccionado com cera utilidade e palito abaixador de língua na forma dos arcos dentários. No intervalo de um ano as mesmas crianças foram remoldadas afim de se verificar se houveram ou não mudanças nas dimensões do arco dentário decíduo. A partir da obtenção dos modelos em gesso, foram realizadas medições por meio de um dispositivo digitalizador tridimensional denominado MicroScribe-3DX nos instantes inicial (primeira moldagem) e final (moldagem após um ano). Foram avaliadas medidas referentes às distâncias inter-segundos molares, interprimeiros molares, intercaninos, perímetro, comprimento de arco e espaços primatas. Consideraram-se ainda dimorfismo sexual, tipo de arco e influência de hábitos. Concluiu-se que as dimensões transversais sofreram aumento significativo na dentadura decídua, enquanto que o perímetro, o comprimento e os espaços primatas permaneceram constantes. As distâncias intermolares apresentaram dimorfismo sexual, com dimensões maiores no gênero feminino. Com relação às medidas de perímetro, comprimento e espaços primatas não ocorreram diferenças significantes em relação ao gênero. As dimensões de comprimento nos arcos não diferem nos arcos decíduos Tipo I e Tipo II de Baume, enquanto que o diâmetro dos arcos Tipo I de Baume, são maiores do que os do Tipo II. Os hábitos de sucção de dedo e chupeta não provocaram alterações nas dimensões dos arcos decíduos no período observado, de um ano.

Palavras-chave: Dentição decídua. Má oclusão. Mudanças dimensionais - pré-escolares.

\section{INTRODUÇÃO E REVISÃO DE LITERATURA}

A má oclusão na dentadura decídua tem sido pouco estudada, parte por dificuldades de acessar e analisar os pacientes de pouca idade e em parte porque esta população, por razões de dificuldades de atendimento, não têm sido alvo do interesse clínico.
É importante conhecer a situação da oclusão dentária de crianças de pequena idade para que medidas de prevenção ou tratamento possam ser planejados a nível institucional de maneira que governos, universidades e instituições de serviços sociais possam estar interessados em informações a respeito da saúde bucal.

* Resumo da Dissertação de Mestrado apresentada à Faculdade de Odontologia de Araraquara - UNESP - abril de 2002

** Mestranda do Curso de Pós-Graduação em Odontologia, da Faculdade de Odontologia de Araraquara - UNESP.

*** Professor Assistente Doutor do Departamento de Clínica Infantil, da Faculdade de Odontologia de Araraquara - UNESP.

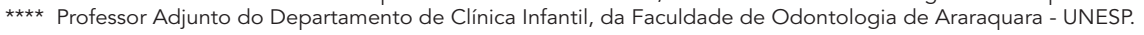


As primeiras observações a respeito da epidemiologia das más oclusões na dentição decídua surgiram no ano de 1915 por Chiavaro ${ }^{5}$. Nesse estudo foi observado que $28,9 \%$ das crianças italianas na faixa etária de 3 a 6 anos já apresentavam algum distúrbio no desenvolvimento da oclusão dentária.

Outros trabalhos ilustram e quantificam a ocorrência das más oclusões na primeira dentição. Os dados provenientes de vários países demonstram variabilidade na prevalência das más oclusões, sendo que, alguns trabalhos revelam uma ocorrência abaixo de $20 \%$; outros demonstram uma ocorrência entre 35 a 55\%; enquanto há ainda alguns que apontam para níveis de prevalência acima de 60\%. No Brasil estudos realizados na dentadura decídua, como de $\mathrm{Maia}^{8}$ na cidade de Natal, no estado do Rio Grande do Norte; e de Brandão et al. ${ }^{4}$, na cidade de Belém, no Estado do Pará; revelaram que aproximadamente 1 de cada 2 crianças apresentavam, já nesta fase do desenvolvimento oclusal, algum tipo de má oclusão. Em 1984 na cidade de São Paulo, Mathias ${ }^{10}$ estudou a má oclusão em crianças com dentadura decídua encontrando uma porcentagem de 79,3\%. Novamente enfatiza-se a importância do conhecimento, bem como a situação da oclusão dentária da população em idades bem precoces para obter indicadores direcionados à aplicabilidade de medidas preventivas.

Em 1952, Barrow e White ${ }^{2}$ estudaram as mudanças nos arcos maxilar e mandibular. Utilizaram 51 crianças, obtendo 528 dados de moldagens seriadas. As moldagens foram feitas em intervalos de um ano com cera e vazadas em gesso para a dentição decídua, e hidrocolóide e vazadas em gesso para a dentição permanente. Os modelos foram examinados individualmente. A largura do arco dental na região de caninos foi obtida pela medida da distância entre as pontas de cúspides em cada arco. Observou-se que houve pequena mudança na largura intercaninos dos 3 aos 5 anos, aumentando rapidamente dos 5 aos 9 anos de idade. A largura do arco na região dos molares foi tomada na ranhura junto à fossa central. Em geral dos 5 aos 10 anos de idade, a largura do arco maxilar e mandibular na região dos segundos molares decíduos aumentou aproximadamente $1,5 \mathrm{~mm}$, ocorrendo a diminuição da largura do arco em somente 7\% dos 51 casos.

Usberti $^{13}$ realizou um estudo morfológico do arco dentário decíduo em crianças na faixa etária de 3 a 6 anos, antes da erupção do primeiro molar permanente. Examinou 1.191 crianças, sendo 483 da cidade de São Paulo - SP e 708 da cidade de Piracicaba - SP. Das crianças examinadas, apenas 120 foram utilizadas no estudo tendo como características: 1) dentadura decídua completa; 2) ausência de cárie ou restaurações interproximais; 3) ausência de má oclusão; 4) ausência de anomalias dentais de forma, número, estrutura e erupção e 5) crianças caucasóides. As crianças foram agrupadas por idade e por gênero, sem distinção entre as cidades. Nesse estudo avaliou-se o tipo de arco, a relação terminal dos molares decíduos, o comprimento total do arco, o comprimento do segmento anterior do arco, o espaço primata, a distância intercanina e a distância intermolar. Para obtenção dessas medidas, utilizou os seguintes dispositivos: 1) compasso tridimensional ortodôntico, modelo Korkhaus, para medir o comprimento total do arco, o segmento anterior do arco e a distância intermolar; 2) compasso de ponta seca da marca Kerm para medir a distância intercanina; 3) paquímetro da marca Mitutoyo com a mesma finalidade; 4) régua milimetrada, adaptada para medir o trespasse horizontal e vertical inter-incisal; 5) fios de aço inoxidável calibrados de 0,3 a 2,6 $\mathrm{mm}$ de diâmetro para medir os espaços primatas e o tipo de arco; 6) espelho clínico com uma lanterna, tipo lápis, adaptada para evidenciação da relação terminal dos molares decíduos. Todas as medidas foram obtidas diretamente da boca das crianças. Em suas conclusões apresentou que, tanto em crianças do gênero masculino como do gênero feminino, o arco tipo I é mais freqüente que o arco tipo II; na faixa etária de 3 a 4 anos predomina a relação 
terminal de segundos molares decíduos em plano, quando comparada ao degrau mesial para mandíbula; com a idade começa a ocorrer um aumento na relação terminal em degrau mesial; o comprimento total do arco decíduo não sofre modificações evidentes em ambos os gêneros; a distância intercanina diminui levemente com a idade em ambos os gêneros; a distância intermolar aumenta levemente com a idade nos indivíduos portadores de arco tipo I e diminui também levemente nos indivíduos portadores de arco tipo II.

Por meio de estudo longitudinal, Leighton e Feasby $^{7}$ avaliaram os fatores que influenciam o desenvolvimento da oclusão molar. Nesse estudo foram considerados 35 casos de Classe I, associandoos com alterações da relação molar sagital durante a infância. Esses casos foram estudados em duas fases: entre a dentição decídua e mista, e entre a dentição mista e permanente. Enfatizaram que na primeira fase, entre as dentições decídua e mista a média de alterações é pequena, mas representa mudanças positivas e negativas das oclusões de canino e molar nos casos individuais e que as mudanças foram algumas vezes assimétricas e acompanhadas por desvio da linha média.

Bishara et $\mathrm{al.}^{3}$, estudando as mudanças da largura intercaninos e intermolares num estudo longitudinal, avaliaram 28 crianças do gênero masculino e 33 do gênero feminino com aproximadamente 6 semanas, 1 ano e 2 anos de idade antes da completa erupção da dentição decídua; e 15 pacientes do gênero masculino e 15 do gênero feminino nas idades de 3, 5, 8, 13, 26 e 45 anos. Os pacientes foram moldados e foram confeccionados modelos de gesso, 5 pontos no arco maxilar e 7 pontos no arco mandibular foram identificados. As medidas de largura maxilar e mandibular nos modelos foram obtidas independentemente por dois examinadores e a confiabilidade foi determinada a $0.5 \mathrm{~mm}$. Dentre as principais conclusões, assinalaram que entre 6 semanas e 2 anos de idade houve um aumento significante da largura posterior e anterior dos arcos maxilares e mandibulares em ambos os gêneros; as larguras intercaninas e intermolares aumentaram significantemente entre 3 e 13 anos de idade em ambos os arcos maxilar e mandibular.

O presente trabalho foi desenvolvido visando a fornecer informações para o melhor entendimento da etiologia, prevalência e características das más oclusões na dentadura decídua em crianças, que freqüentam as creches da Prefeitura Municipal da cidade de Araraquara, no Estado de São Paulo. Deste modo, a natureza dessa pesquisa fundamenta-se no estudo anual em crianças de pequena idade.

\section{MATERIAL E MÉTODO Seleção da amostra}

Foram avaliadas crianças leucodermas, com idade variando de 3 a 5 anos e meio. Essas crianças freqüentavam a pré-escola da Rede Pública do Município de Araraquara, sendo visitadas dezoito creches.

A seleção das crianças para a amostra foi realizada de acordo com os seguintes procedimentos:

Inicialmente foi obtida a lista de nomes das crianças de cada uma das salas de aula, contendo gênero e idade. Foram selecionados 25\% dos pré-escolares de cada sala de aula, sendo metade pertencente ao gênero masculino e a outra metade ao gênero feminino. Para essa finalidade, utilizou-se um critério de seleção sistemático, em que a primeira criança da lista era selecionada, contava-se quatro crianças e a quinta também era selecionada, até que se completassem os $25 \%$ dos pré-escolares propostos acima. Caso a lista de chamada não completasse os $25 \%$, reiniciaria a seleção pelo segundo nome da lista e se repetia o mesmo procedimento de contar quatro crianças, selecionando a quinta para fazer parte da amostra.

O volume amostral para o desenvolvimento do projeto, bem como os ajustes da metodologia empregada foram estabelecidos por meio de um projeto experimental piloto. 
Utilizando-se esse processo foram selecionados 838 pré-escolares, mas somente 535 deles foram submetidos ao processo de tomada de registro da superfície oclusal dos dentes com o dispositivo especialmente construído para tal, e os 303 préescolares restantes, não tiveram força ou coordenação para realizar o procedimento de morder a cera.

Após um ano, as mesmas crianças foram novamente submetidas ao processo de tomada de registro da superfície oclusal dos dentes, porém a amostra reduziu-se para 235 pré-escolares, pois 300 destas crianças não se encontravam nas suas respectivas creches ou cidade.

\section{Tomada de registro da superfície oclusal dos dentes}

Para a realização da tomada de registro da superfície oclusal dos dentes foi utilizada cera utilidade e palito abaixador de língua descartável. A cera foi recortada, segundo a forma dos arcos dentários, em duas partes, superior e inferior, sendo que entre elas foi acoplado o palito abaixador de língua, preconizado por Martins et al..$^{9}$ (Fig. 1) .

O objetivo do palito abaixador foi facilitar a introdução do conjunto na boca da criança e mantê-lo em posição durante o registro. A seguir, o conjunto foi colocado em posição, solicitando a criança mordê-lo até que as cúspides dos dentes superiores e inferiores ficassem registradas na cera. $\mathrm{Na}$ seqüência, o conjunto foi retirado da boca e imerso em água na temperatura normal, para prevenir possíveis distorções.

\section{Obtenção dos modelos}

A partir do dispositivo confeccionado em cera utilidade foram obtidos dois modelos, um correspondente ao arco superior e outro ao arco inferior.

Esses modelos foram obtidos em gesso comum a partir das marcas digitais oclusais das arcadas superior e inferior registradas na cera, e banhadas em álcool e, secas com jato de ar para a remoção de qualquer impureza, evitando assim algum tipo de alteração no gesso.

O gesso comum foi vertido nas marcas digitais oclusais e aguardou-se a presa do mesmo para que os modelos pudessem ser removidos do conjunto de cera. Vazou-se com gesso comum primeiro um lado do dispositivo e, após a obtenção do modelo referente a este lado, procedeu-se ao vazamento do outro lado. A seguir, os mesmos foram recortados.

\section{Obtenção das medidas}

Os modelos de gesso, contendo o registro da superfície oclusal dos dentes decíduos, foram utilizados para obtenção das informações sobre os

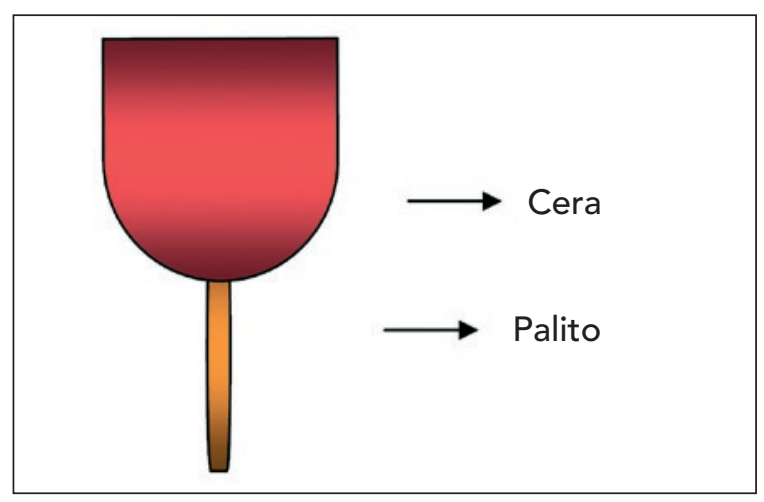

FIGURA 1 - Dispositivo para registro da superfície oclusal dos dentes.

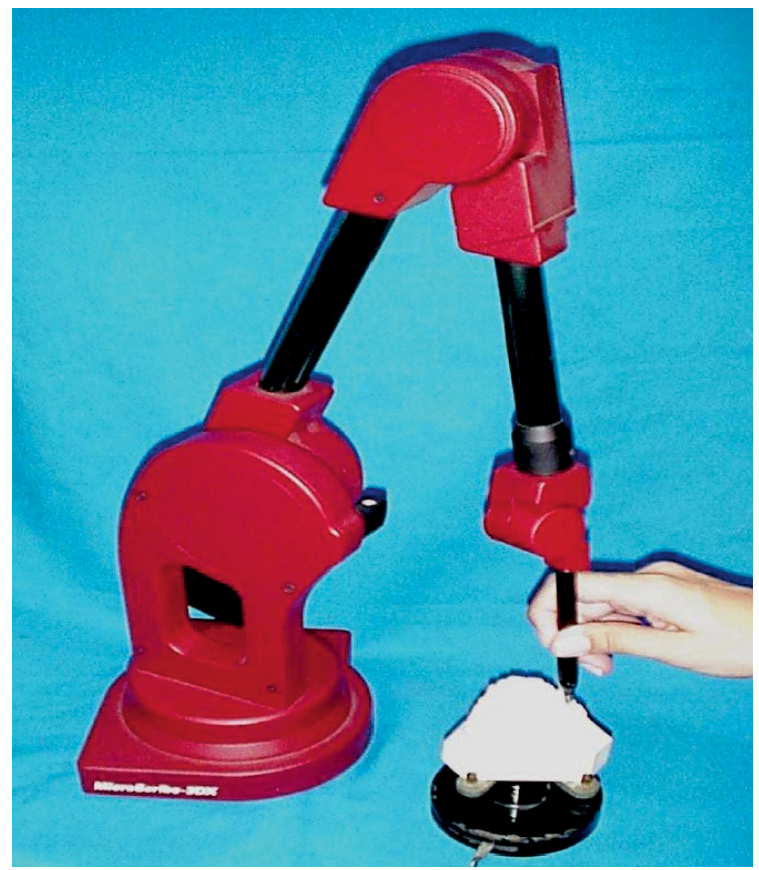

FIGURA 2 - Digitalizador MicroScribe - 3DX. 


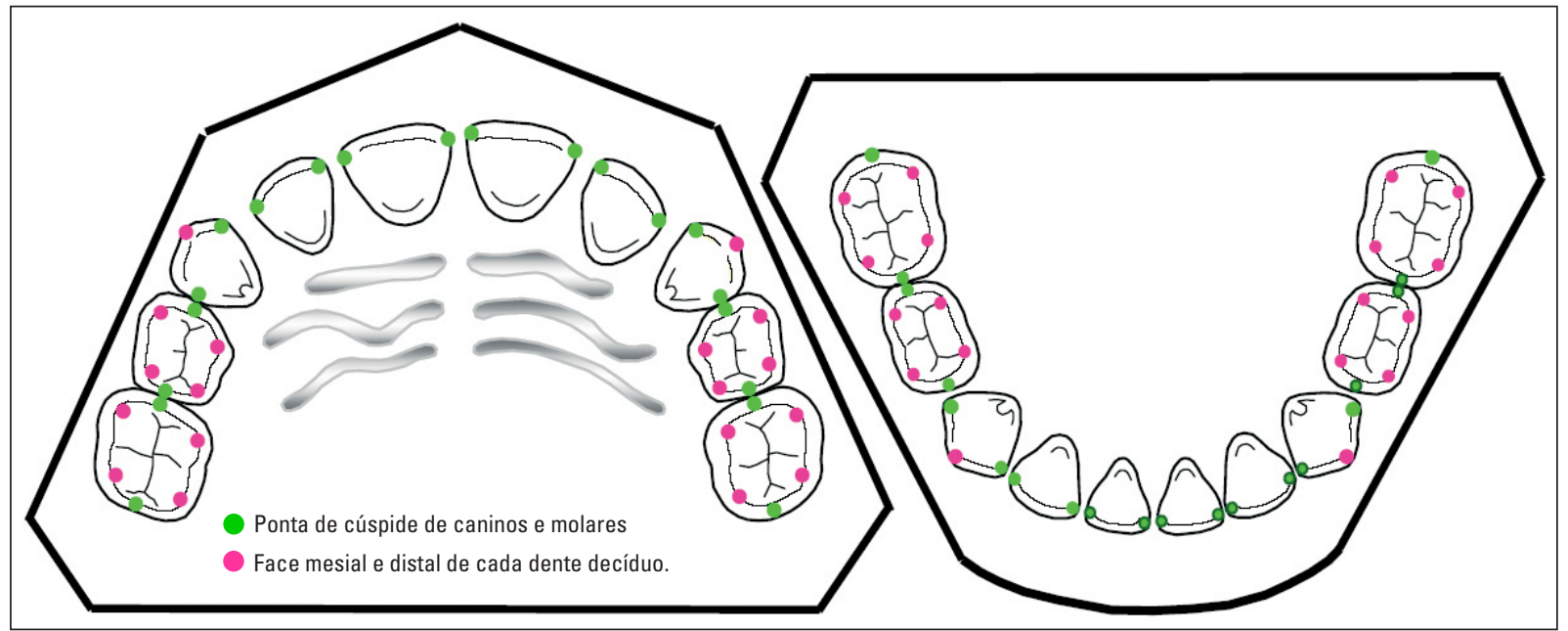

FIGURA 3 - Pontos digitalizados

arcos superior e inferior das crianças. Para cada arco, superior ou inferior, foram determinadas dimensões intermolares, intercaninos, perímetro e comprimento do arco, e também a medida do espaço primata dos lados direito e esquerdo.

Para obtenção dessas medidas, utilizou-se um aparelho digitalizador tridimensional, denominado MicroScribe-3DX (Fig. 2), acoplado a um computador. Foram digitalizados pontos pré-determinados nos modelos de gesso superior e inferior (Fig. 3).

Os pontos digitalizados foram registrados na forma de coordenadas X, Y e Z numa planilha do programa Excel da Microsoft, especialmente desenvolvido para essa finalidade.

\section{RESULTADO}

Os valores obtidos nas mensurações dos modelos em gesso foram submetidos à estatística teste t-Student, F de Snedecor e probabilidade.

Com relação às mudanças das dimensões dos arcos dentários as distâncias intermolares e intercaninos sofreram aumento significante no período de um ano. O perímetro do arco inferior também mostrou aumento siginificante. Tratando-se de dimorfismo sexual houveram diferenças estatisiticamente significantes nas distâncias intermolares em relação ao gênero feminino. Para a variável Tipo de arco, as mesmas medidas citadas acima também mostram diferenças significntes em relação ao arco Tipo II de Baume, o qual exibe dimensões maiores que os do Tipo I.

\section{DISCUSSÃO}

Deste modo, resultados referentes à avaliação das mudanças das dimensões transversais estão concordantes com as conclusões de Barrow e White ${ }^{2}$ em um estudo semelhante, anteriormente realizado. Do mesmo modo, as moldagens foram feitas em intervalos de um ano e vazadas em gesso. Nas conclusões obtidas argumentaram que houve pequena mudança na largura intercaninos dos 3 aos 5 anos de idade. Os valores obtidos no presente estudo, variaram de $1,11 \mathrm{~mm}$ para o arco superior e 1,18 $\mathrm{mm}$ para o arco inferior (Fig. 4). Para a distância inter-segundos molares decíduos, os autores encontraram valores de aumento aproximado de 1,5 mm, enquanto que os resultados desta pesquisa evidenciaram valores da ordem de $0,78 \mathrm{~mm}$ para o arco superior e $1,1 \mathrm{~mm}$ para o arco inferior.

Os presentes resultados também estão de acordo com aqueles encontrados por $\mathrm{Knott}^{6}$, que relatou aumento de $3 \mathrm{~mm}$ na região de primeiros molares decíduos, enquanto que os valores médios encontrados neste trabalho foram de $3,07 \mathrm{~mm}$ 
para o arco superior.

Essas informações coincidem com as conclusões de Bishara et al. ${ }^{3}$, que, estudando as mudanças nas distâncias intercaninos e intermolares em estudo longitudinal, informaram que entre 6 semanas e 2 anos de idade ocorreram aumentos significantes das larguras posteriores dos arcos maxilar e mandibular em ambos os gêneros, enquanto que as larguras intercaninos aumentaram

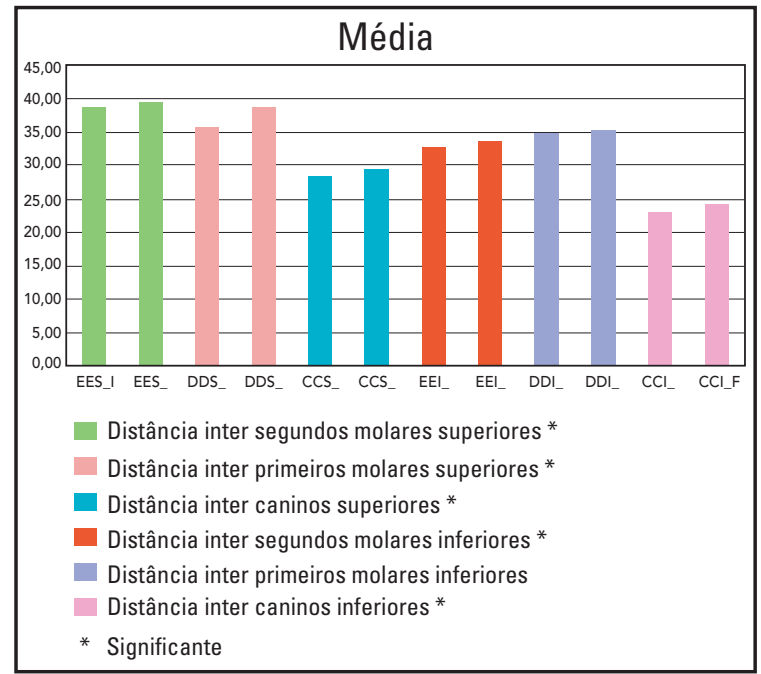

FIGURA 4 - Avaliação das mudanças das dimensões transversais dos arcos dentários nos instantes inicial e final.

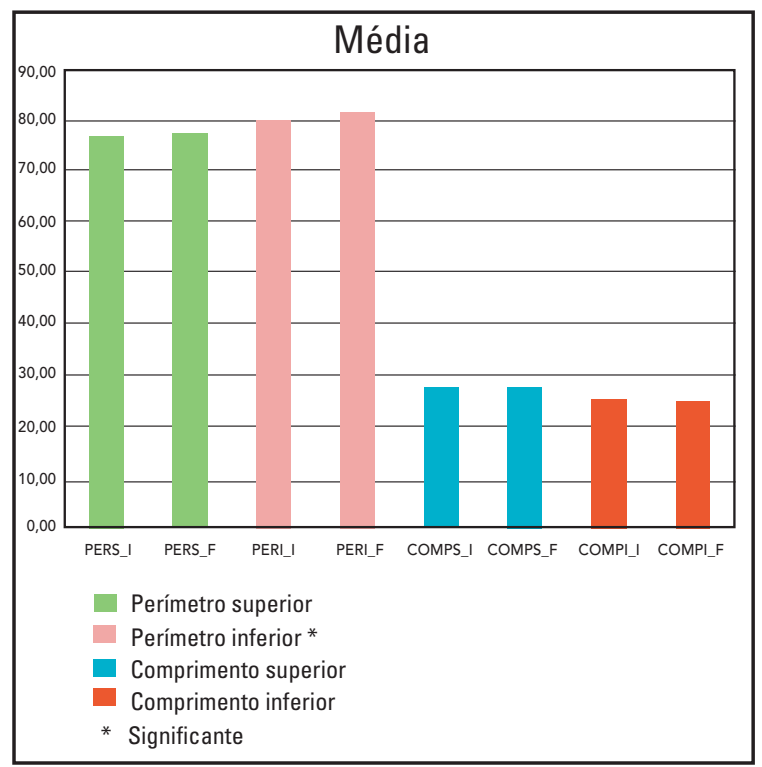

FIGURA 5 - Avaliação das mudanças das dimensões de perímetro e comprimento dos arcos dentários nos instantes inicial e final significantemente entre 3 e 13 anos de idade em ambos os arcos maxilar e mandibular.

Os presentes resultados estão de acordo com Moyers ${ }^{11}$ que assinala que, em termos de desenvolvimento da oclusão, o perímetro do arco constitui a dimensão mais crítica, pois não pode aumentar durante o crescimento e começa a diminuir com a idade. Os resultados encontrados, embora expressem alguma significância, não apresentam relevância clínica,

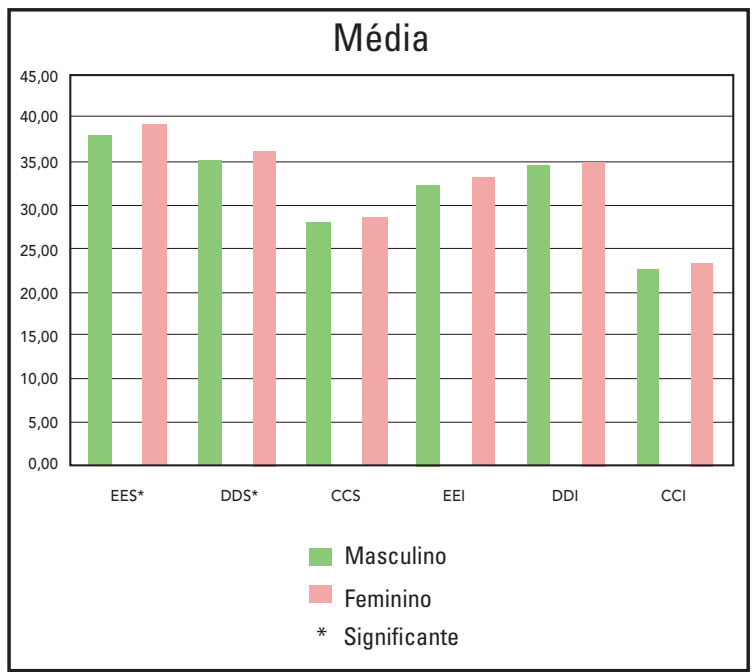

FIGURA 6 - Distâncias inter-segundos molares, interprimeiros molares e intercaninos segundo gênero no instante inicial.

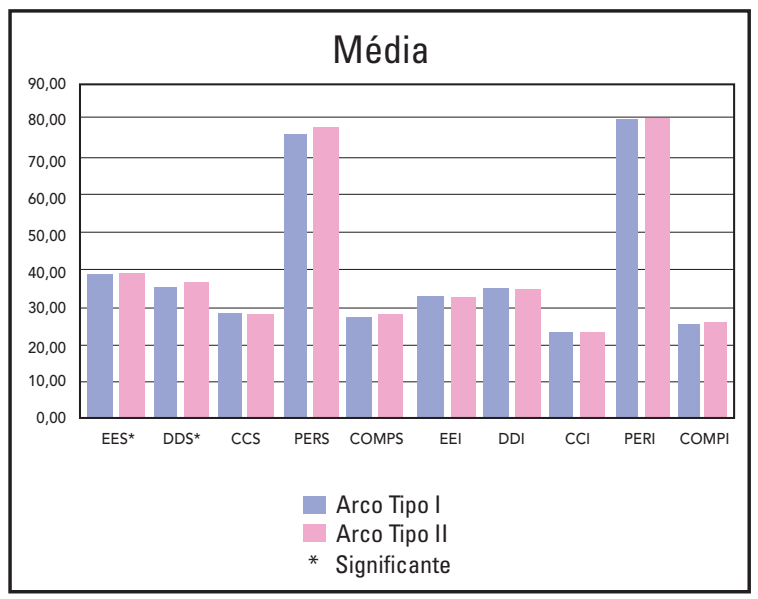

FIGURA 7 - Medidas transversais, perímetro e comprimento de arco segundo tipo de arco no instante inicial. 
tendo em vista as diferenças encontradas (Fig. 5).

O dimorfismo sexual mais evidente em relação ao gênero feminino, talvez possa ser explicado pelo fato das meninas se desenvolverem mais rápido e apresentarem um grau de maturidade mais avançado (Fig. 6).

Com relação a Tipo de arco, os resultados obtidos discordam parcialmente das opiniões emitidas por Almeida ${ }^{1}$, quando assinala que os arcos superiores do Tipo I, apresentam as médias dos lados maiores que os do Tipo II e entretanto concordam que os arcos inferiores possuem dimensões menores que a dos arcos superiores e também que o comprimento do arco superior e inferior não diferem nos arcos Tipo I e Tipo II de Baume. Por outro lado, nossos resultados concordam plenamente com as conclusões de Ohno et al. ${ }^{12}$, afirmam que os diâmetros dos arcos Tipo I são maiores que os do Tipo II de Baume ( Fig. 7).

\section{CONCLUSÃO}

De acordo com a avaliação longitudinal, as dimensões transversais sofreram aumento significativo na dentadura decídua, enquanto que o perímetro, o comprimento e os espaços primatas permaneceram constantes.

As distâncias interprimeiros molares e intersegundos molares apresentaram dimorfismo sexual, apresentando dimensões maiores no gênero feminino.

O diâmetro dos arcos dentários decíduos do Tipo I de Baume é maior que os do Tipo II.

\title{
Changes in dental arches dimensions of 3 to 6 year old children
}

\begin{abstract}
Two hundred and thirty five children enrolled in kindergarden of Araraquara public school system were evalueted and cast models were obtained. For this purpose a special apparatus was confeccioned with utility wax and exam stick following the dental arch form. After an year same children were evaluated again in order to observe possible changes in the primary dental arches. Using the cast models some measurements were acquired by a tridimensional digitalizer, MicroScribe-3DX, on the first and after one year cast models. Second molars, first molars and canine width, arch perimeter, arch length and primate space were evaluated. Gender dimorphism, arch type and finger or pacifier sucking habits were also considered. It was concluded that the transverse measurements showed a significant increase in primary dentition, while the perimeter, arch length and primate spaces were stable. The first and second molars width presented gender dimorphism, with higher values in the females. Considering the perimeter, arch length and primate spaces there were no significant differences according to gender. The arch length dimensions were not different between Baume Type I and II arches, althougth Baume Type I arch width were larger than Type II. Finger and pacifier sucking habits had no effect on primary dentition dimensions during the observed period of one year.
\end{abstract}

Key words: Primary dentition. Malocclusion. Dimensions changes - kindergarden. 


\section{REFERÊNCIAS}

1. ALMEIDA, M. Estudo da morfologia do arco dentário decíduo, tipo I e II de Baume, pela geometria analítica: contribuição para seu estudo. 1976. 53 f. Dissertação (Mestrado em Odontopediatria)-Faculdade de Odontologia, Universidade de São Paulo, São Paulo, 1976.

2. BARROW, G. V.; WHITE, J. R. Developmetal changes of the maxillary and mandibular dental arch. Angle Orthod, Appleton, v. 22, no.1, p. 41-48, 1952.

3. BISHARA, S. et al. Arch width changes from 6 weeks to 45 years of age. Am J Orthod Dentofacial Orthop, St. Louis, v. 111, p. $401-409,1997$

4. BRANDÃO, A. M. M. et al. Oclusão normal e má oclusão na dentição decídua: um estudo epidemiológico em pré-escolares do município de Belém - PA. Rev Paran Odontol, Belém, v.1 n.1, p. 13-7, 1996

5. CHIAVARO, A. Malocclusion of temporary teeth. Int J Orthod Oral Surg, St. Louis, v. 1, p.171-179, 1916.

6. KNOTT, V. B. Longitudinal study of dental arch widths at four stages of dentition. Angle Orthod, Appleton, v. 42, p. 387394, 1972.

7. LEIGHTON, B. C.; FEASBY, W. H. Factors influencing the development of molar occlusion: A longitudinal study. Br J Orthod, London, v.15, p. 99-103, 1988.

8. MAIA, N. G. Prevalência de más oclusões em pré - escolares da cidade de Natal, na fase de dentição decídua. 1987. 86 f. Tese (Mestrado em Odontologia Social)- Faculdade de Odontologia, Universidade Federal do Rio Grande do Norte, Natal, 1987.
9. MARTINS, J. C. R. et al. Prevalência de má oclusão em pré-escolares de Araraquara: relação da dentição decídua com hábitos e nível sócio-econômico. R Dental Press Ortodon Ortop Facial, Maringá, v. 3, n. 6, p. 35-43, 1998.

10. MATHIAS, R. S. Prevalência de algumas anomalias de oclusão na dentadura decídua: relação terminal desfavorável dos segundos molares decíduos, mordida aberta anterior, apinhamento anterior e mordida cruzada posterior. 1984. 56 f. Dissertação (Mestrado em Odontopediatria)-Faculdade de Odontologia, Universidade de São Paulo, São Paulo, 1984.

11. MOYERS, R. Development of occlusion. Dent Clin North Am, Philadelphia v. 13, p. 523- 536, 1969.

12. OHNO, N.; KASHIMA, K.; SAKAI, T. A study on interdental spaces of the deciduos dental arch in Indian sample. Aichi Gakuin Daigaku Shigakkai Shi, Nagoya, v. 28, p. 79-91, 1990.

13. USBERTI, A. C. Estudo morfológico do arco dentário decíduo em crianças na faixa etária de $\mathbf{3}$ a $\mathbf{6}$ anos, antes da erupção dos primeiros molares permanentes. 1979. $81 \mathrm{f}$.Tese (LivreDocência em Odontopediatria)-Faculdade de Odontologia, Universidade Estadual de Campinas, Piracicaba, 1979.
Endereço para correspondência

Taís Cristina dos Santos Dinelli

Rua Antonio Rodrigues Cajado, 1390

13560-380 - São Carlos/SP

Email : dinelli@uol.com.br 